generally grow quickly, shortening the generation time and conferring on them a relatively faster evolutionary change that supports their prosperity on our planet. One of the key organs involved in the rapid growth from any type of food is the intestinal tract. The intestinal tract, the gut, is one of the largest organs in animals, including insects, implying its importance. The intestinal tract functions to store ingested food, digest food for extraction of nutrients to be absorbed, and excrete undigested residue. Peristalsis, a radially symmetrical contraction and relaxation of muscles that propagates in a wave down the intestinal tract, is controlled by motor neurons (Furness, 2012; Olsson and Holmgren, 2001), essentially aiding digestion processes. Food ingestion and its amount in the gut is sharply recognized by sensory neurons: stretching of the gastrointestinal muscle activates the mechanosensory receptors expressed in the muscle cells, transmitting the signals to the neurons (Furness, 2012). Such information is reflected to the secretion of digestive juices, which include degrading enzymes from the gut epithelia and digestive glands.

In mammals, $70 \%$ of neurons in the body are estimated to reside in the intestine. Auerbach's plexus provides motor innervation to the muscular layer, controlling mainly peristalsis, and also has some sensory roles (Baumgarten et al., 1970; Furness, 2012), whereas Meissner's plexus provides secretomotor innervation to the mucosa nearest the intestinal lumen, governing the secretion of digestive enzymes as well as ciliary movement of the epithelia (Goyal and Hirano, 1996). The endocrine system also modulates gut functions. Incretins, gastrointestinal peptide hormones secreted by the sensing of glucose and fat in the intestine, stimulate an increase in the release of insulin from the pancreas and slow the nutrient absorption rate into the bloodstream by reducing gastric emptying, which may directly reduce food intake (Baggio and Drucker, 2007). Then, blood sugar concentration is sensed again by the intestinal cells. Low glucose levels and an empty stomach stimulate the release of the peptide hormone ghrelin from specific cells in the gastrointestinal tract. Ghrelin acts in the central nervous system to induce appetite and feeding behaviors, as well as to increase gastric acid secretion and gastrointestinal motility to prepare the body for food intake (Kojima and Kangawa, 2005; Nakazato et al., 2001). Thus, the intestinal tract plays the role of a command center for controlling energy homeostasis.

These gastrointestinal roles of the nervous and enteroendocrine systems are well studied in mammals, but relatively less is known in insects except for the control of peristalsis (Donini et al., 2002; French et al., 2014; Lange and Chan, 2008; Robertson et al., 2012) and peptide hormone secretion (Dircksen et al., 2008; McCormick and Nichols, 1993; Palmer et al., 2007; Price et al., 2002). In particular, although D. melanogaster is utilized as a model animal for investigating developmental processes and innate immune responses, studies of gut physiology have been less prevalent (Dow, 2003). In the last few years, however, several important findings relating to the role of enteric neurons and the function and structure of the Drosophila intestinal tract have been reported. In this review, we first briefly explain the structure and function of the Drosophila gut, and then summarize recent reports on the functions of fly enteric neurons. For more information on the endocrine control of gut function, 
please refer to other reviews (Veenstra et al., 2008).

\section{Structure and function of the Drosophila gut}

The Drosophila intestinal tract comprises a monolayer of epithelial cells, minor populations of intestinal stem cells and enteroendocrine cells, and surrounding visceral muscles (Buchon et al., 2013a; Kuraishi et al., 2013). Motor neurons and sensory neurons innervate the muscles and epithelia of the digestive tract (Lemaitre and Miguel-Aliaga, 2013). A semipermeable membrane called the peritrophic matrix lines the lumen (Hegedus et al., 2009; Lehane, 1997). The gut is divided into the foregut, midgut, and hindgut, based on the developmental origin. Ingested foods are stored in the crop (only in adults), a part of the foregut, and then pushed into the midgut by the pumping action of the proventriculus. The midgut is the main region of digestion and absorption of the ingested foods, and is further subdivided into several regions based on histological and functional differences (Buchon et al., 2013b). The hindgut is responsible for the absorption of water, salts, and other beneficial substances before excretion. Blind-ended ducts called Malpighian tubules, which are attached at the anterior end of the hindgut, are the main organs of osmoregulation and excretion (Dow et al., 1994). Cells of the intestinal tract require oxygen. Fine chitinous tubes called trachea deliver oxygen and expel carbon dioxide directly to and from the tissues (Samakovlis et al., 1996). Some trachea attach to the gut and branch off into smaller tubes called tracheoles, which surround the intestinal tract.

The peritrophic matrix is an acellular structure that forms a layer comprising chitin polymers and glycoproteins such as peritrophins, protecting the midgut epithelium from abrasive food and microbes (Kuraishi et al., 2013). The peritrophic matrix is thought to be produced in the proventriculus in Drosophila adults. Though structurally different, the peritrophic matrix is considered analogous to the mucous layer of the mammalian digestive tract. In Drosophila adults, drosocrystallin (dcy) is a structural component of the peritrophic matrix, and a loss-of-function mutation of $d c y$ causes reduced thickness of the peritrophic matrix and higher permeability, and is associated with higher susceptibility to ingested entomopathogenic bacteria or pore-forming toxins (Kuraishi et al., 2011).

Digestive enzymes and transporters are required for digestion and absorption, respectively, in the midgut. Bioinformatic analysis revealed the presence of many digestive enzymes including carbohydrases, lipases, proteinases, and lysozymes, and transporters such as glucose and ion transporters (Buchon et al., 2013b). Only a few biochemical or genetic functional studies of these molecules, however, have been performed to date. The expression of digestive enzymes differs between each of the midgut regions, indicating sequential processing of ingested foods along the intestine, and this regionalization involves the peritrophic matrix as it attaches to many proteins and is considered to be where digestive processes occur (Hegedus et al., 2009). With regard to the transcriptional regulation of digestive enzymes, a recent report revealed that the expression of carbohydrases and lipase is suppressed in Drosophila adults fed a glucose-rich diet (Chng et al., 2014). High glucose levels in the hemolymph, the insect blood, stimulate the secretion of transforming growth factor $\beta$ (TGF- $\beta$ ) from the fat body, an insect 
equivalent of the mammalian liver. The TGF- $\beta$ activates TGF- $\beta$ /Activin signaling in the epithelial cells of the midgut, resulting in the repression of digestive enzymes. Insulin signaling is well known to be involved in sugar metabolism, and this study demonstrated that TGF- $\beta$ signaling also plays an important role in metabolism.

The intestinal epithelium is frequently exposed to harmful pathogens, and thus must be armed with an efficient and powerful immune system to protect itself (Buchon et al., 2013a; Kuraishi et al., 2013). Currently two resistance mechanisms against invading microbes are known: production of reactive oxygen species and secretion of antimicrobial peptides into the hemolymph. Dual oxidase, Duox, is a plasma membrane-expressing NADPH oxidase involved in the production of reactive oxygen species in the gut (Bae et al., 2010). Pathogenic bacteria such as Erwinia carotovora carotovora 15 (Ecc15) release uracil that activates an as-yet unidentified $\mathrm{G}$ protein-coupled receptor, leading to the enzymatic activation of Duox through the $\mathrm{G}$ protein alpha subunit q protein $(\mathrm{G} \alpha \mathrm{q})$ - phospholipase $\mathrm{C} \beta$ (PLC $\beta$ ) signaling pathway (Ha et al., 2009a; Lee et al., 2013). Upon severe infection, Duox expression is upregulated through triggering the MEKK1/MEK3/p38 pathway, which activates activating transcription factor 2 (ATF2) thereby mediating Duox transcription (Ha et al., 2009b). Antimicrobial peptides can damage and kill microbes, and their expression is under the control of the immunodeficiency (Imd) pathway (Buchon et al., 2009; Ryu et al., 2008; Tzou et al., 2000). Pattern recognition receptor peptidoglycan recognition protein (PGRP)-LE and PGRP-LC sense Gram-negative bacteria (Bosco-Drayon et al., 2012; Neyen et al., 2012), then activate the Imd pathway, which induces nuclear translocation of the NF- $\kappa \mathrm{B}$ transcriptional factor Relish that is responsible for the expression of antimicrobial peptide genes (Lemaitre and Hoffmann, 2007).

\section{Innervation of neurons in the Drosophila intestinal tract}

For simplification, in this review, we use the term "enteric neurons" to refer to neurons with neurites extending into the proventriculus, the midgut, and the hindgut. In Drosophila larvae, intestinal innervations are observed in the proventriculus, anterior and posterior midgut, and the hindgut (Hartenstein et al., 1994; Schoofs et al., 2014; Spiess et al., 2008). These neurites are innervated from the ventral ganglion and the proventricular ganglion, which are located near the ventral nerve cord and the proventriculus, respectively. From those ganglions, further innervations are sent to the pharynx, esophagus, the ring gland, and the central nervous system, comprising a complex stomatogastric nervous system (Hartenstein et al., 1994; Schoofs et al., 2014). Anatomical details of the stomatogastric nervous system is relatively less understood in Drosophila compared to large insects (Braunig, 2008; Copenhaver, 2007; Hartenstein, 1997; Rand and Ayali, 2010). Most larval enteric neurons are thought to be motor neurons that direct the movement of intestinal visceral muscles, yet, as described in the next section, there is also sensory innervation in the larval intestine, at least at the posterior part of the hindgut. Thanks to the rich library of enhancer trap GAL4 lines of Drosophila, enteric neurons are classified on the basis of specific GAL4-positive criteria (Table 1, Fig. 1). TRH (Tryptophan hydroxylase)-GAL4-positive serotonergic neurons innervate the proventriculus 
and the anterior midgut (Schoofs et al., 2014), Ilp7 (Insulin-like-peptide 7)-GAL4-positive neurons (Miguel-Aliaga et al., 2008) and PDF (Pigment-dispersing factor)-GAL4-positive neurons innervate the hindgut (Talsma and Christov, 2012), while HGN1 (Hypogastric nerves-1)-GAL4-positive neurons innervate the posterior hindgut (Zhang et al., 2014).

In Drosophila adults, the proventriculus, the anterior midgut, and the hindgut apparently receive innervation from the brain, the ventral ganglion, and the proventricular ganglion (Cognigni et al., 2011). For the regulation of peristaltic movement of the intestinal tract, the majority of the innervation is likely to be efferent, although the existence of dendrites indicates sensory innervation in the gut (Cognigni et al., 2011). With respect to specific GAL4 positivity, motor neuron driver OK371-GAL4-positive neurons and sensory neuron drivers ppk1.9-GAL4 and cha7.4-GAL4-positive neurons innervate the proventriculus and the hindgut (Cognigni et al., 2011). Muscles and epithelial cells of the proventriculus and the anterior midgut receive innervation from NP3253-GAL4-positive neurons (Kenmoku et al., manuscripts in revision). Insulin-peptide-producing neurons Ilp2- and Ilp7-positive neurons innervate the proventriculus and the hindgut, while GMR51F12-GAL4 and GMR48A05-GAL4-positive neurons innervate the proventriculus and the anterior midgut (Cognigni et al., 2011; Olds and Xu, 2014). The hindgut receives innervation from HGN1-, PDF- and odd-GAL4-positive neurons (Cognigni et al., 2011; Talsma and Christov, 2012; Olds and $\mathrm{Xu}, 2014)$.

\begin{tabular}{llll} 
Larvae & GAL4 & Tissue & Rreferences \\
\hline & TRH-GAL4 & proventriculus, anterior midgut & Schoofs et al., 2014 \\
\hline Ilp7-GAL4 & hindgut & Miguel-Aliaga et al., 2008 \\
\hline PDF-GAL4 & hindgut & Talsma and Christov, 2012 \\
\hline HGN1-GAL4 & posterior hindgut & Zhang et al., 2014 \\
Adult & & \\
\hline GAL4 & Tissue & Rreferences \\
\hline OK371-GAL4 & proventriculus, hindgut & Cognigni et al., 2011 \\
\hline ppk1.9-GAL4 & proventriculus, hindgut & Cognigni et al., 2011 \\
\hline cha7.4-GAL4 & proventriculus, hindgut & Cognigni et al., 2011 \\
\hline NP3253-GAL4 & proventriculus, anterior midgut & Kenmoku et al., manuscripts in revision \\
\hline Ilp2-GAL4 & proventriculus, hindgut & Cognigni et al., 2011 \\
\hline Ilp7-GAL4 & proventriculus, hindgut & Cognigni et al., 2011 \\
\hline GMR51F12-GAL4 & proventriculus, anterior midgut & Olds and Xu, 2014 \\
\hline GMR48A05-GAL4 & proventriculus, anterior midgut & Olds and Xu, 2014 \\
\hline HGN1-GAL4 & hindgut & Cognigni et al., 2011 \\
\hline odd-GAL4 & hindgut & Olds and Xu, 2014 \\
\hline PDF-GAL4 & hindgut & Talsma and Christov, 2012
\end{tabular}

\section{Role of enteric neurons in feeding and excretion behavior}

4.1. Feeding and excretion assays

In Drosophila larvae, feeding and excretion are simply assessed using dye mixed 
with fly food, and the behavior is evaluated by observing the amount of color in the intestine or feces (Zhang et al., 2014). In Drosophila adults, a capillary feeder (CAFE) assay is considered to be a standard method to analyze feeding behavior (Ja et al., 2007). The CAFE assay can be used to evaluate the amount of meal volume and the frequency of ingestion by recording the consumption of liquid-dyed food contained in a glass capillary. To analyze defecation, Cognigni et al established a quantitative and semi-automatic protocol using a lipid probe that only becomes fluorescent after exposure to the fly's digestive tract (Cognigni et al., 2011). They named this probe Fluoropoo, which was used to supplement standard fly food for the assay. The total amount of feces produced can be easily quantified by this "Fluoropoo assay". Further, the addition of the $\mathrm{pH}$ indicator dye bromophenol blue (BPB) to the food with Fluoropoo provides information about the size, shape, concentration, and color of the excreta. The Fluoropoo assay also predicts food intake with appropriate controls.

\subsection{Feeding behavior}

Ilp-producing neurons are involved in feeding behavior in Drosophila adults. Suppression of Ilp7-GAL4-positive neurons results in an increased food intake, revealed by the CAFE and Fluoropoo assays (Cognigni et al., 2011; Olds and Xu, 2014). In parallel, the suppression leads to a high glucose concentration in the hemolymph of adult flies. Degenerin/epithelial sodium channels (DEG/ENaCs) are evolutionally conserved mechanosensory ion channels that sense mechanical tension and generate action potentials in expressed neurons (Hwang et al., 2007; Zhong et al., 2010). Pickpocket 1 (PPK1) belongs to the $\mathrm{DEG} / \mathrm{ENaCs}$ family of proteins that functions in Ilp7-GAL4-positive neurons to sense satiety to foods. PPK1 repression by RNAi-mediated knockdown or pharmacological inhibition phenocopies the suppression of Ilp7-GAL4-positive neurons, i.e., resulting in increased food intake (Olds and $\mathrm{Xu}, 2014)$. On the other hand, suppression of Ilp2-GAL4-positive median neurosecretory cells (mNSCs), which synapse with Ilp7-GAL4-positive neurons at the ventral ganglion and innervate the foregut, results in decreased food intake. Taken together, these findings suggest that PPK1-expressing Ilp7-GAL4-positive neurons sense the extension of the hindgut by ingested food, and transmit the information to the mNSCs, which negatively regulate the appetite of the Drosophila adult.

\subsection{Defecation behavior}

Drosophila larvae display defecation cycles of stereotypic frequency. Glutaminergic PDF-GAL4-positive and HGN1-GAL4-positive motor neurons are involved in sequential contraction of the hindgut and anal sphincter to regulate this defecation cycle (Zhang et al., 2014). These subsets of neurons form functional synapses and exhibit a periodic firing pattern that is synchronized with the hindgut contraction. The opening of the anus is accompanied by defecation. The single anal sensory neuron, the PPK (pickpocket)-GAL4-positive neuron that innervates the anal sphincter and senses the stretch when the anal slit opens, provides feedback information to the PDF- and HGN1-GAL4-positive neurons. A mechanosensory ion channel, NOMPC (no mechanoreceptor potential C), is involved in the sensing of the radial stretch of the anal sphincter in the anal sensory neuron (Zhang et al., 2014). 


\section{Interaction of enteric neurons with neighboring organs}

\subsection{Malpighian tubules}

The hindgut-innervating PDF-GAL4-positive neurons are considered to derive from a neuroendocrine cell lineage that produces the peptide hormone PDF (Siga, 2003; Veenstra et al., 2008). To analyze the visceral role of PDF, Talsma et al evaluated the similarity of PDF with the mammalian peptide hormone vasoactive intestinal polypeptide (VIP) (Larsson et al., 1976). VIP is a gut-brain hormone that acts on the G-protein coupled VIP receptor to mediate the contraction of smooth muscles of the intestinal or urinary tracts. They found that ex vivo treatment of the adult intestinal tract with PDF does not cause peristalsis of the midgut or hindgut, but interestingly, induces contraction of the Malpighian tubules (Talsma and Christov, 2012). The Malpighian tubules are a functional equivalent of the mammalian urinary organ, and are involved in detoxification and stress sensing (Davies et al., 2014). Muscles surrounding the tubules express PDF receptors. PDF-dependent contraction of the tubule muscles ex vivo is dependent on PDF receptors expressed in the muscles. Artificial activation of gut-innervating PDF neurons induces contractions of the tubules in vivo. These findings suggest that enteric neurons control neighboring organs, the Malpighian tubules, through peptide hormone secretion (Talsma and Christov, 2012).

\subsection{Trachea}

Insect respiration is accomplished without lungs. Instead, oxygen is delivered directly to tissues through the tracheal system, which comprises holes called spiracles on the insect exoskeleton that allow air to enter tubes (trachea), which are invaginations of the cuticular exoskeleton that branch throughout the body, including the intestinal tract. The smallest tubes, tracheoles, penetrate cells and serve as diffusion sites for water, oxygen, and carbon dioxide. This system could be considered an alternative to mammalian blood vessels, in that the formation of capillary networks is an adaptive process mainly controlled by the metabolic needs of target tissues (Fraisl et al., 2009). Indeed, Linneweber et al found that tracheal branching, which is regulated by the proliferation of tracheal terminal cells, in the gut is sensitive to a reduction in nutrient availability (Linneweber et al., 2014). They observed that fibroblast growth factor signaling, a well-known signaling pathway that promotes tracheal branching in response to hypoxia, is not involved in nutrition-dependent tracheal plasticity. Rather, insulin signaling, a major coordinator of nutrient intake and tissue size in many animals, is responsible for regulating tracheal terminal cell growth in a cell-autonomous manner. Anatomically, posterior tracheal branches are close to hindgut innervating neurons that are Ilp7- and PDF-GAL4-positive. In fact, they showed that these neurons are activated by food intake. Furthermore, a gain-of-function experiment revealed that Transient receptor potential A1 (TrpA1)-mediated artificial activation of these cells promotes tracheal branching in the posterior hindgut. These findings indicate that enteric neurons regulate the intestinal trachea in response to the host metabolic status (Linneweber et al., 2014) 
6. Relevance of enteric neurons to the peritrophic matrix and midgut structure

Kenmoku et al performed genetic screening to identify neurons important to gut integrity using GAL4 enhancer trap lines with an Inward rectifier $\mathrm{K}(+)$ channel Kir2.1 to block neural activity (Kenmoku et al, manuscript in revision). They identified a subset of enteric neurons, NP3253-GAL4-positive neurons, that are required for gut barrier function. Nerve fibers of NP3253-GAL4-positive neurons are observed in the brain, crop, proventriculus, and anterior midgut, and their cell bodies locate in the brain and the most anterior part of the midgut. A large number of these neurons seems to innervate the gut visceral muscles, but some innervate the epithelia. Inhibition of NP3253-GAL4-positive neurons enhances the susceptibility of flies to oral infection with Erwinia carotovora carotovora 15 (Ecc15), a Gram-negative bacterium. Oral infection with green fluorescent protein (GFP)-labeled Ecc15 revealed led to observation of the GFP signal throughout the body of the NP3253-inhibited flies in contrast to wild-type flies that retained the GFP signal only in their abdomen.

The permeability of the peritrophic matrix is an important feature for the barrier function of the gut, which is assessed by feeding adults fluorescein isothiocyanate (FITC)-labeled particles. Particles that are not permeable to the wild-type peritrophic matrix are diffused in the gut of NP3253-inhibited flies upon feeding. Intriguingly, an altered morphology of the proventriculus and the anterior midgut, e.g., a widened lumen of the R1 region of the midgut is observed when NP3253-GAL4-positive neurons are inhibited in adults after 1-2 days post emergence. A bromophenol blue (BPB) feeding experiment showed that region-specific $\mathrm{pH}$ is disrupted in the NP3253-inhibited midgut. On the other hand, feeding and defecation behaviors of the NP3253-inhibited flies are normal. Artificial activation of the NP3253-GAL4-positive neurons does not induce any obvious phenotypes. The study by Kenmoku et al revealed a new role for enteric neurons in the homeostatic control of gut integrity, including the permeability of the physical barrier of the intestinal tract (Fig. 2).

Some questions remain: which subset of neurons in the NP3253-GAL4-positive cells is responsible for maintaining the structural integrity of the gut?; which cells in the gut are innervated?; and what are the molecular mechanisms of this neural control? One possibility is that NP3253-GAL4-positive neurons control the production and secretion of the peritrophic matrix in the proventriculus. If the peritrophic matrix is impaired, epithelial cells of the midgut would be exposed to the commensal bacteria and toxic compounds in the food. This could explain the leaky midgut and susceptibility of the NP3253-inhibited flies to oral infection with pathogenic bacteria. It is also possible that NP3253-GAL4-positive neurons govern peristaltic movement of the gut, particularly that of the proventriculus. If the pumping action of the proventriculus is compromised, the secreted peritrophic matrix may not form a proper layer throughout the gut, resulting in malfunction of the gut barrier. Another possibility is that if the function of the visceral muscles is impaired, the midgut structure would be loosened as observed in NP3253-inhibited flies. Further studies are needed to assess the roles of NP3253-GAL4-positive neurons and the underlying molecular mechanisms. 


\section{Conclusion}

In this review, we describe the involvement of enteric neurons in many aspects of gut structure and function, as well as in neighboring organs, such as the trachea and the Malpighian tubules. Compared to studies in large insects, the anatomical characterization of enteric neurons in Drosophila is just beginning. Further, the roles of enteric neurons in Drosophila intestinal physiology are relatively scantly analyzed in comparison with the roles in mammals. Little is known about how enteric neurons contribute to the secretion of digestive enzymes and the absorption of nutrients in the Drosophila intestine. The relationship between enteric neurons and the gut defense system in flies has not yet been analyzed (Sharkey and Savidge, 2014). Due to the wide array of available genetic techniques such the GAL4-UAS system, however, some analyses in Drosophila have already surpassed those in other animals. These studies were the first to demonstrate in animals the requirement for enteric neurons for maintenance of the physical gut barrier and control of the plastic vascular structure by sensing the metabolic status of the host. Future studies in D. melanogaster are expected to reveal the mechanisms underlying the enteric neuronal control of intestinal physiology, and to provide new concepts regarding the neuronal control of host homeostasis.

\section{Acknowledgements}

This work was supported by grants from the Japan Science and Technology Agency (JST); the Ministry of Education, Culture, Sports, Science and Technology of Japan (MEXT); the Kao Foundation for Arts and Sciences; the Uehara Memorial Foundation; and Futaba Electronics Memorial Foundation.

\section{Author contributions}

T. K. and H. K. wrote the draft. H. K. drew the pictures. T. K. managed the writing. T. K., H. K., and S. K. completed the manuscript.

The authors declare no conflict of interest.

\section{References}

Bae, Y.S., Choi, M.K., and Lee, W.J. (2010). Dual oxidase in mucosal immunity and host-microbe homeostasis. Trends Immunol 31, 278-287.

Baggio, L.L., and Drucker, D.J. (2007). Biology of incretins: GLP-1 and GIP. Gastroenterology 132, 2131-2157.

Baumgarten, H.G., Holstein, A.F., and Owman, C. (1970). Auerbach's plexus of mammals and man: electron microscopic identification of three different types of neuronal processes in myenteric ganglia of the large intestine 
Bosco-Drayon, V., Poidevin, M., Boneca, I.G., Narbonne-Reveau, K., Royet, J., and Charroux, B. (2012). Peptidoglycan sensing by the receptor PGRP-LE in the Drosophila gut induces immune responses to infectious bacteria and tolerance to microbiota. Cell Host Microbe 12, 153-165.

Braunig, P. (2008). Neuronal connections between central and enteric nervous system in the locust, Locusta migratoria. Cell Tissue Res 333, 159-168.

Buchon, N., Broderick, N.A., Chakrabarti, S., and Lemaitre, B. (2009). Invasive and indigenous microbiota impact intestinal stem cell activity through multiple pathways in Drosophila. Genes Dev 23, 2333-2344. Drosophila melanogaster. Nat Rev Microbiol 11, 615-626.

Buchon, N., Osman, D., David, F.P., Fang, H.Y., Boquete, J.P., Deplancke, B., and Lemaitre, B. (2013b). Morphological and molecular characterization of adult midgut compartmentalization in Drosophila. Cell Rep 3, $1725-1738$. 
Dircksen, H., Tesfai, L.K., Albus, C., and Nassel, D.R. (2008). Ion transport peptide splice forms in central and

366 peripheral neurons throughout postembryogenesis of Drosophila melanogaster. J Comp Neurol 509, 23-41.

367

368

369

370

Donini, A., Ngo, C., and Lange, A.B. (2002). Evidence for crustacean cardioactive peptide-like innervation of the gut in Locusta migratoria. Peptides 23, 1915-1923.

Dow, J.A. (2003). The Drosophila phenotype gap - and how to close it. Brief Funct Genomic Proteomic 2, $121-127$

Dow, J.A., Maddrell, S.H., Gortz, A., Skaer, N.J., Brogan, S., and Kaiser, K. (1994). The malpighian tubules of Drosophila melanogaster: a novel phenotype for studies of fluid secretion and its control. J Exp Biol 197, $421-428$.

Fraisl, P., Mazzone, M., Schmidt, T., and Carmeliet, P. (2009). Regulation of angiogenesis by oxygen and metabolism. Dev Cell 16, 167-179.

French, A.S., Simcock, K.L., Rolke, D., Gartside, S.E., Blenau, W., and Wright, G.A. (2014). The role of serotonin in feeding and gut contractions in the honeybee. J Insect Physiol 61, 8-15.

Furness, J.B. (2012). The enteric nervous system and neurogastroenterology. Nat Rev Gastroenterol Hepatol 9, 286-294.

Goyal, R.K., and Hirano, I. (1996). The enteric nervous system. N Engl J Med 334, 1106-1115.

Ha, E.M., Lee, K.A., Park, S.H., Kim, S.H., Nam, H.J., Lee, H.Y., Kang, D., and Lee, W.J. (2009a). Regulation of DUOX by the Galphaq-phospholipase Cbeta-Ca2+ pathway in Drosophila gut immunity. Dev Cell 16, 386-397.

Ha, E.M., Lee, K.A., Seo, Y.Y., Kim, S.H., Lim, J.H., Oh, B.H., Kim, J., and Lee, W.J. (2009b). Coordination of multiple dual oxidase-regulatory pathways in responses to commensal and infectious microbes in drosophila gut. Nat Immunol 10, 949-957.

Hartenstein, V. (1997). Development of the insect stomatogastric nervous system. Trends Neurosci 20, 421-427. 
Hartenstein, V., Tepass, U., and Gruszynski-Defeo, E. (1994). Embryonic development of the stomatogastric nervous system in Drosophila. J Comp Neurol 350, 367-381.

Hegedus, D., Erlandson, M., Gillott, C., and Toprak, U. (2009). New insights into peritrophic matrix synthesis, architecture, and function. Annu Rev Entomol 54, 285-302.

Hwang, R.Y., Zhong, L., Xu, Y., Johnson, T., Zhang, F., Deisseroth, K., and Tracey, W.D. (2007). Nociceptive neurons protect Drosophila larvae from parasitoid wasps. Curr Biol 17, 2105-2116.

Ja, W.W., Carvalho, G.B., Mak, E.M., de la Rosa, N.N., Fang, A.Y., Liong, J.C., Brummel, T., and Benzer, S. (2007). Prandiology of Drosophila and the CAFE assay. Proc Natl Acad Sci U S A 104, 8253-8256.

Kenmoku, H., Ishikawa, H., Ote, M., Kuraishi, T., and Kurata, S. A subset of enteric neurons controls the permiability of the peritrophic matrix and midgut structure in Drosophila adults. (manuscripts in revision)

Kojima, M., and Kangawa, K. (2005). Ghrelin: structure and function. Physiol Rev 85, 495-522.

Kuraishi, T., Binggeli, O., Opota, O., Buchon, N., and Lemaitre, B. (2011). Genetic evidence for a protective role of the peritrophic matrix against intestinal bacterial infection in Drosophila melanogaster. Proc Natl Acad Sci U S A $108,15966-15971$.

Kuraishi, T., Hori, A., and Kurata, S. (2013). Host-microbe interactions in the gut of Drosophila melanogaster. Front Physiol 4, 375.

Lange, A.B., and Chan, K. (2008). Dopaminergic control of foregut contractions in Locusta migratoria. J Insect Physiol 54, 222-230.

Larsson, L.I., Fahrenkrug, J., Schaffalitzky De Muckadell, O., Sundler, F., Hakanson, R., and Rehfeld, J.R.

(1976). Localization of vasoactive intestinal polypeptide (VIP) to central and peripheral neurons. Proc Natl Acad Sci U S A 73, 3197-3200.

Lee, K.A., Kim, S.H., Kim, E.K., Ha, E.M., You, H., Kim, B., Kim, M.J., Kwon, Y., Ryu, J.H., and Lee, W.J. 
$410 \quad$ Cell 153, 797-811.

411 Lehane, M.J. (1997). Peritrophic matrix structure and function. Annu Rev Entomol 42, 525-550.

412 Lemaitre, B., and Hoffmann, J. (2007). The host defense of Drosophila melanogaster. Annu Rev Immunol 25, $413 \quad 697-743$

Lemaitre, B., and Miguel-Aliaga, I. (2013). The digestive tract of Drosophila melanogaster. Annual review of 415 genetics 47, 377-404.

Linneweber, G.A., Jacobson, J., Busch, K.E., Hudry, B., Christov, C.P., Dormann, D., Yuan, M., Otani, T., Knust, E., de Bono, M., et al. (2014). Neuronal control of metabolism through nutrient-dependent modulation of tracheal branching. Cell 156, 69-83.

McCormick, J., and Nichols, R. (1993). Spatial and temporal expression identify dromyosuppressin as a brain-gut peptide in Drosophila melanogaster. J Comp Neurol 338, 278-288.

Miguel-Aliaga, I., Thor, S., and Gould, A.P. (2008). Postmitotic specification of Drosophila insulinergic neurons from pioneer neurons. PLoS Biol 6, e58.

Nakazato, M., Murakami, N., Date, Y., Kojima, M., Matsuo, H., Kangawa, K., and Matsukura, S. (2001). A role for ghrelin in the central regulation of feeding. Nature 409, 194-198.

Neyen, C., Poidevin, M., Roussel, A., and Lemaitre, B. (2012). Tissue- and ligand-specific sensing of gram-negative infection in drosophila by PGRP-LC isoforms and PGRP-LE. J Immunol 189, 1886-1897.

Olds, W.H., and Xu, T. (2014). Regulation of food intake by mechanosensory ion channels in enteric neurons. eLife 3. 128, 481-503. 
Palmer, G.C., Tran, T., Duttlinger, A., and Nichols, R. (2007). The drosulfakinin 0 (DSK 0) peptide encoded in the conserved Dsk gene affects adult Drosophila melanogaster crop contractions. J Insect Physiol 53, 1125-1133.

Price, M.D., Merte, J., Nichols, R., Koladich, P.M., Tobe, S.S., and Bendena, W.G. (2002). Drosophila melanogaster flatline encodes a myotropin orthologue to Manduca sexta allatostatin. Peptides 23, 787-794.

Rand, D., and Ayali, A. (2010). Neuroanatomy and neurophysiology of the locust hypocerebral ganglion. J Insect Physiol 56, 884-892.

Robertson, L., Rodriguez, E.P., and Lange, A.B. (2012). The neural and peptidergic control of gut contraction in Locusta migratoria: the effect of an FGLa/AST. J Exp Biol 215, 3394-3402.

Ryu, J.H., Kim, S.H., Lee, H.Y., Bai, J.Y., Nam, Y.D., Bae, J.W., Lee, D.G., Shin, S.C., Ha, E.M., and Lee, W.J. (2008). Innate immune homeostasis by the homeobox gene caudal and commensal-gut mutualism in Drosophila. Science 319, 777-782.

Samakovlis, C., Hacohen, N., Manning, G., Sutherland, D.C., Guillemin, K., and Krasnow, M.A. (1996).

Development of the Drosophila tracheal system occurs by a series of morphologically distinct but genetically coupled branching events. Development 122, 1395-1407.

Schoofs, A., Huckesfeld, S., Surendran, S., and Pankratz, M.J. (2014). Serotonergic pathways in the Drosophila larval enteric nervous system. J Insect Physiol 69, 118-125.

Sharkey, K.A., and Savidge, T.C. (2014). Role of enteric neurotransmission in host defense and protection of the gastrointestinal tract. Auton Neurosci 181, 94-106.

Siga, S. (2003). Anatomy and functions of brain neurosecretory cells in diptera. Microsc Res Tech 62, 114-131.

Spiess, R., Schoofs, A., and Heinzel, H.G. (2008). Anatomy of the stomatogastric nervous system associated with the foregut in Drosophila melanogaster and Calliphora vicina third instar larvae. J Morphol 269, 272-282.

Talsma, A.D., and Christov, C.P. (2012). Remote control of renal physiology by the intestinal neuropeptide 
Tzou, P., Ohresser, S., Ferrandon, D., Capovilla, M., Reichhart, J.M., Lemaitre, B., Hoffmann, J.A., and Imler,

J.L. (2000). Tissue-specific inducible expression of antimicrobial peptide genes in Drosophila surface epithelia. Immunity 13, 737-748.

Veenstra, J.A., Agricola, H.J., and Sellami, A. (2008). Regulatory peptides in fruit fly midgut. Cell Tissue Res 334, 499-516.

Zhang, W., Yan, Z., Li, B., Jan, L.Y., and Jan, Y.N. (2014). Identification of motor neurons and a mechanosensitive sensory neuron in the defecation circuitry of Drosophila larvae. eLife 3.

Zhong, L., Hwang, R.Y., and Tracey, W.D. (2010). Pickpocket is a DEG/ENaC protein required for mechanical nociception in Drosophila larvae. Curr Biol 20, 429-434.

Figure Legends

Figure 1 Illustration summarizing the innervation of the larval and adult intestinal tract.

In larvae, TRH-GAL4-positive neurons (yellow) innervate the proventriculus and the anterior midgut, PDF-GAL4-positive neurons (red) and Ilp7-GAL4-positive neurons (brown) innervate the hindgut, and HGN1-GAL4-positive neurons (blue) innervate the posterior hindgut. In adults, Ilp2-GAL4-positive neurons (brown) innervate the proventriculus; NP3253-GAL4-positive neurons (red) project to the proventriculus, crop, and the anterior midgut; and GMR48A05-GAL4 (yellow) and GMR51F12-GAL4 (orange) positive neurons project to the proventriculus and the anterior midgut. The hindgut receives innervations from Ilp7-, HGN1-, odd- and PDF-GAL4-positive neurons. Cell bodies are represented by circles. $\mathrm{VNC}$, ventral nerve cord. 
484

492

Figure 2 Presumed model of NP3253-GAL4-positive neuron-dependent gut integrity maintenance.

(A) A lateral view of the proventriculus and anterior midgut of adults.

NP3253-GAL4-positive neurons (green) innervate muscles and epithelial cells of the proventriculus and the anterior midgut. (B) Inhibition of NP3253-GAL4-positive neurons (blue) with Kir2.1 leads to high peritrophic matrix permeability and abnormal gut structure. EC, enterocyte; PM, peritrophic matrix. 


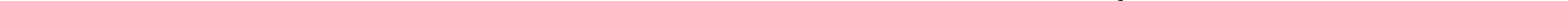


$A$

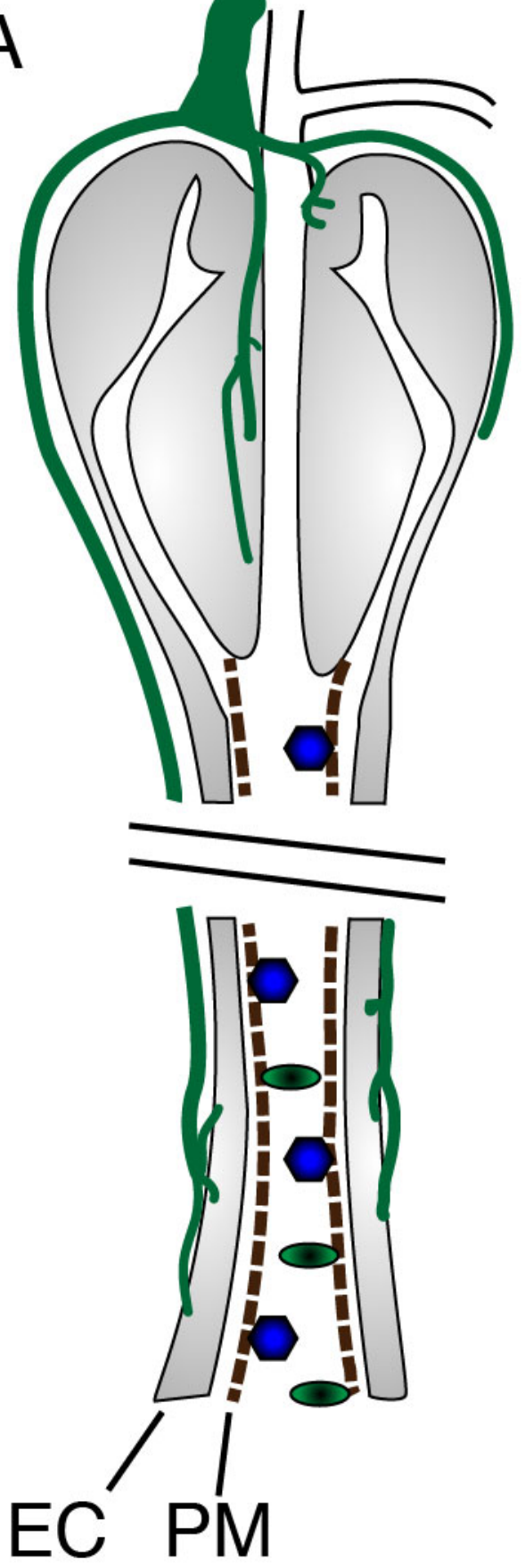

B

Fig 2
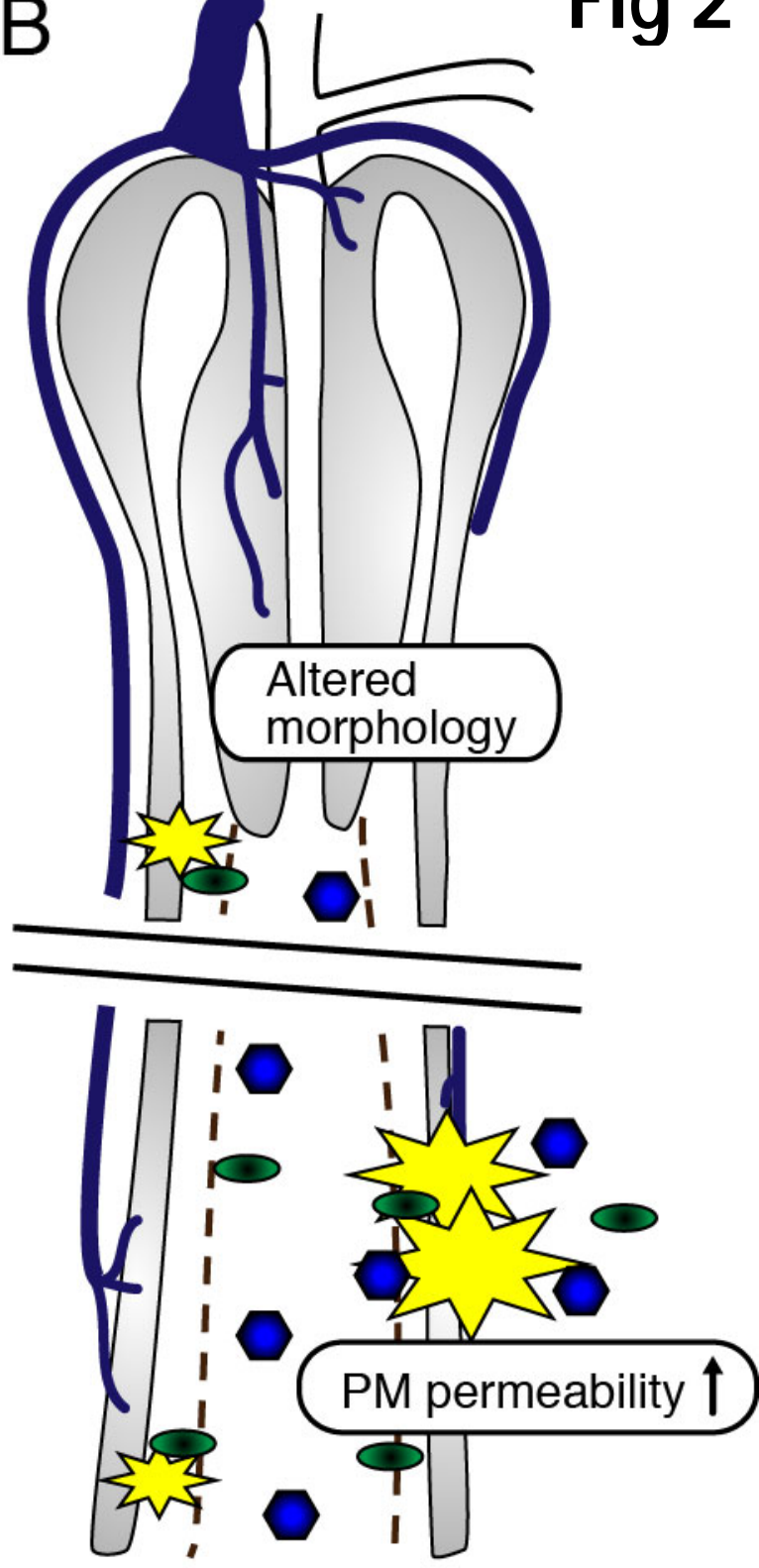
Brain

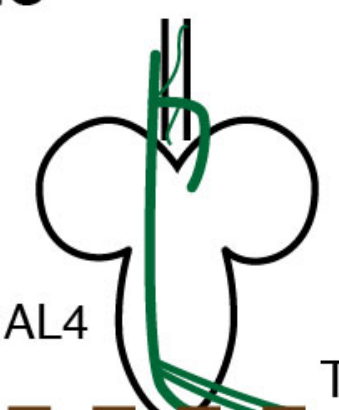

Foregüt ----- To Hindgut

Proventriculus

Midgut

Malpighian tubules

\section{-}

Hindgut

IIp7-GAL4

PDF-GAL4

HGN1-GAL4

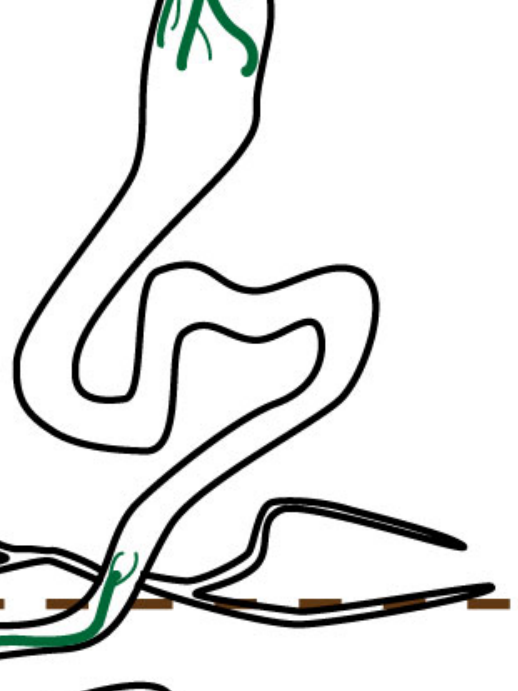

To VNC

To VNC

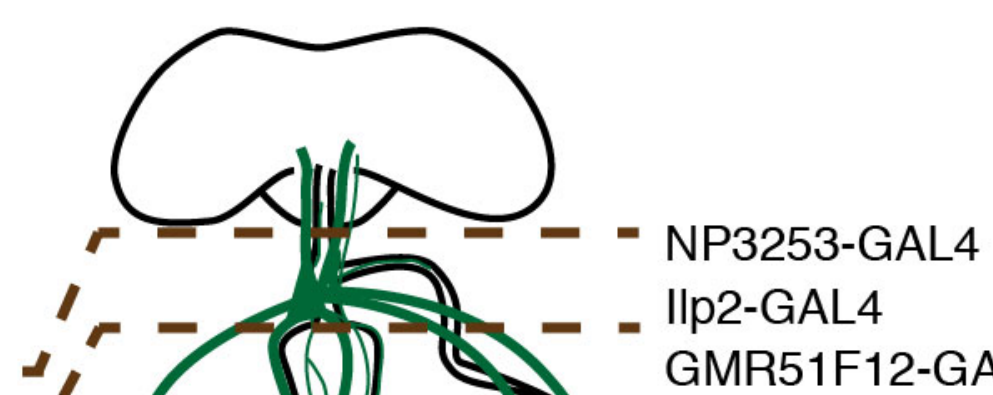

GMR51F12-GAL4 - - GMR48A05-GAL4 